\title{
Investigation of Inlet Boundary Conditions on Capillary Membrane with Porous Wall during Dead-End Backwash
}

Hussam Mansour* and Wojciech Kowalczyk

Chair of Mechanics and Robotics, University of Duisburg-Essen, Lotharstr, Duisburg, Germany

\begin{abstract}
The capillary membrane technology has become one of the effective methods for producing drinking water. The membrane lifetime and permeability are significantly affected by operating and backwash conditions. To enhance the backwash process, the flow in the porous wall and the pressure drop inside the capillary membrane were investigated numerically. For this purpose, 3D model describing steady-state laminar flow inside the capillary membrane operated in dead-end mode was simulated. The influence of various boundary conditions on both the flow pattern inside the capillary membrane and the characteristic of the membrane were studied. Hereby, the pressure drop in the module and the axial as well as radial velocity profile were estimated with the consideration of the membrane fouling. The calculation of permeate flux contributes to increase the backwash performance and minimize energy consumption. The method of coupling Navier-Stokes equation for the free flow and Darcy-Forchheimer approach for the prediction of the flow in the porous membrane is proposed in the current study. The CFD model was validated by comparing the numerical results with the experimental data. A very good agreement was achieved.
\end{abstract}

Keywords: Ultrafiltration; Porous capillary; Dead-end; Backwash; CFD

\section{Nomenclature}

$$
\begin{aligned}
& \text { u: velocity }(\mathrm{m} / \mathrm{s}) \\
& \text { p: pressure }(\mathrm{Pa}) \\
& \text { v: kinematic viscosity }\left(\mathrm{m}^{2} / \mathrm{s}\right) \\
& \mu \text { : dynamic viscosity }(\mathrm{kg} / \mathrm{m} . \mathrm{s}) \\
& \rho: \text { density }\left(\mathrm{kg} / \mathrm{m}^{3}\right) \\
& \text { D: viscous resistance }\left(1 / \mathrm{m}^{2}\right) \\
& \text { F: inertial resistance }(1 / \mathrm{m})
\end{aligned}
$$

\section{Introduction}

Capillary membrane filtration process applied in water treatment makes use of the porous structure of the membrane. For the backwash process operated in dead-end mode, the driving force is generated by exposing the capillary to high outer pressure that forces the water to flow through the porous wall in outside-in direction. The magnitude of the driving force depends on many factors, among others, on the pressure drop in the capillary membrane. Predicting the behavior of the flow adjacent to the porous wall is essential to calculate the pressure drop and loss in the capillary and in the porous wall, respectively. Consequently, the energy consumption can be reduced, the permeate flux can be estimated and the performance of the backwash process can be improved.

The complexity of interaction between the free and porous flow regimes highlights the demand to develop a CFD model which can optimize the backwash operating parameters. Thus, the CFD model provides a library of boundary conditions that predicts the corresponding pressure drop in the capillary and the axial and radial velocity profile with the consideration of the membrane fouling. Furthermore, the model investigates the influence of the membrane characteristic and the operating parameters on the performance of the backwash process.

The flow inside a tube with porous wall has been for long time under investigation. The early models to solve Navier-Stokes equation for a laminar flow in porous tube were proposed in Berman [1] and Yuan [2] and extended by Bernales [3]. Berman [1] presented a solution to the two-dimensional model in laminar flow in channels with uniform wall suction. This study was extended to cylindrical coordinates to investigate suction as well as injection in flow along a porous channel [2]. Also there is a description of the flow in the porous wall for a circular tube with uniform suction [4-6] and for different permeability [7]. Further investigation was performed for a tube with variable wall suction [8]. Karode [9] derived analytical solution for the pressure drop in a rectangular slit and cylindrical tube with porous walls for a constant permeability. This expression was in an agreement with the solution proposed by Berman [1]. Another expression for the pressure drop and velocity was derived by Kim [10]. It is obtained by applying the perturbation theory to a dead-end cylindrical porous tube. More recently [11], one dimensional model is introduced to determine the fluid in a porous channel with wall suction or injection based on the analytical solution proposed by Berman and Yuan $[1,2]$.

Linking Darcy and Stokes equation [12] contributes to understanding of interfacial phenomena in coupled free and porous flow regimes applicable to Cross flow filtration. Various methods for coupling the free flow and the flow in permeable wall models were introduced and the strength and weakness of each method were discussed [13]. An analytical solution for the coupling between the transmembrane pressure and velocity in tubular membrane is proposed and validated with direct numerical simulation [14]. With Stokes

*Corresponding authors: Hussam Mansour, Chair of Mechanics and Robotics, University of Duisburg-Essen, Lotharstr. Duisburg, Germany, Tel: +49 203379 3342; Fax: +49 203379 2494; E-mail: hussam.mansour@uni-due.de

Received September 19, 2015; Accepted November 19, 2015; Published November 26, 2015

Citation: Mansour H, Kowalczyk W (2015) Investigation of Inlet Boundary Conditions on Capillary Membrane with Porous Wall during Dead-End Backwash J Membra Sci Technol 5: 138. doi:10.4172/2155-9589.1000138

Copyright: (c) 2015 Mansour $\mathrm{H}$, et al. This is an open-access article distributed under the terms of the Creative Commons Attribution License, which permits unrestricted use, distribution, and reproduction in any medium, provided the original author and source are credited. 
equation free flow dynamics and with Darcy equation non-isothermal, non-inertial and incompressible flow in a porous medium is modeled. Darcy's law was extended to non-Stokes flow and the resulting relation between pressure gradient and mass flux in porous media is presented [15].

In order to provide better insight into the flow and pressure drop in membrane channel with porous wall, a CFD model was developed which offers more detailed information about the physical phenomena accompanied with filtration process $[16,17]$. Another work predicts the pressure drop and permeates flux in the hollow fiber using a simplified model equation [18].

Therefore, the optimization of the backwash process is associated with developing a numerical model which describes accurately the pressure drop under various boundary conditions and the flow through the porous wall of a capillary membrane in ultrafiltration module. Furthermore, adjusting the correct boundary conditions for further simulations of backwash process needs the knowledge of the velocity distribution in vicinity of the membrane surface.

\section{Governing Equations}

The fluid dynamic behavior of an incompressible, laminar and viscous flow in steady state is described by continuity Equation 1 and momentum Equation 2. The porous wall is simplified as homogeneous and isotropic medium. In order to incorporate the flow through the porous wall in the considered model, a source term $S$ is added to the momentum equation [19]. This term is distributed in two parts, a viscous loss term $\mathrm{D}$ and an inertial loss term $\mathrm{F}$. These two terms represent the properties of the fluid and the porous wall. The pressure drop has a proportional relation to the velocity in the viscous term and squared velocity in the inertial term whose effects become dominant with the increase of the velocity. The viscous parameter is calculated as the inverse of the permeability whereas the inertial term was not considered $(\mathrm{F}=0)$

$$
\begin{aligned}
& \nabla \mathrm{u}=0 \\
& \mathrm{u} \nabla \mathrm{u}=\frac{1}{\rho} \nabla \mathrm{p}+\mathrm{v} \nabla^{2} \mathrm{u}+\mathrm{S} \\
& \mathrm{S}=-\left(\mu \mathrm{D}+\frac{1}{2}|\mathrm{u}| \mathrm{F}\right) \mathrm{u}
\end{aligned}
$$

\section{Numerical Model}

A single phase, steady-state, laminar, incompressible flow in a capillary membrane with a porous wall was carried out (Figure 1). The capillary length is $980 \mathrm{~mm}$ and has an inner and outer diameter of 1.4 $\mathrm{mm}$ and $2.3 \mathrm{~mm}$, respectively. The equations above will be solved in a computational domain discretized in 11 Mio structured control volume. This high mesh resolution covers the whole computational domain and ensures mesh independent solution. Further mesh refinement has no influence on the solution and will only increase computational efforts and time. The element size is uniform distributed in the capillary inside the capillary membrane and can be seen in Figure 2 .

The meshing is generated in ANSYS-ICEM with ${ }^{\star}$ mesh extension and read in OpenFOAM by the command fluentMeshToFoam. RenumberMesh command is used to rearrange the numbering of the cells in all time directories which will reduce the computational efforts.

Once the mesh is generated, the governing equation in the previous

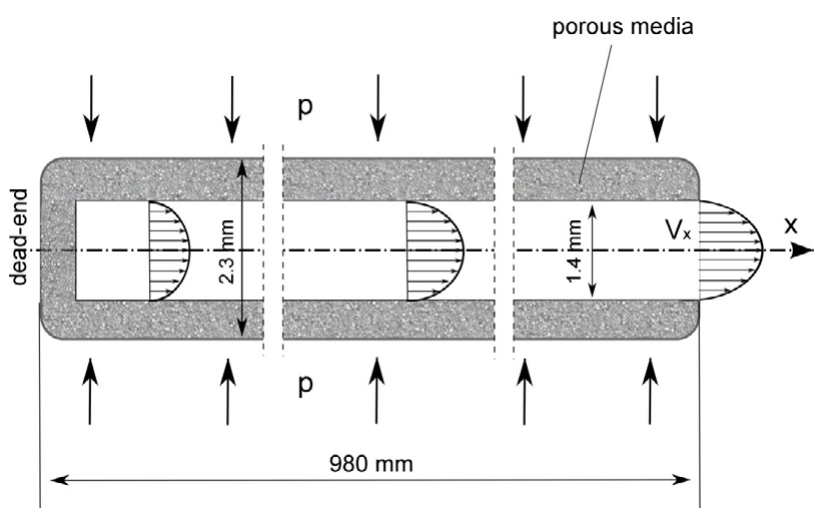

Figure 1: Computational domain of the porous capillary.

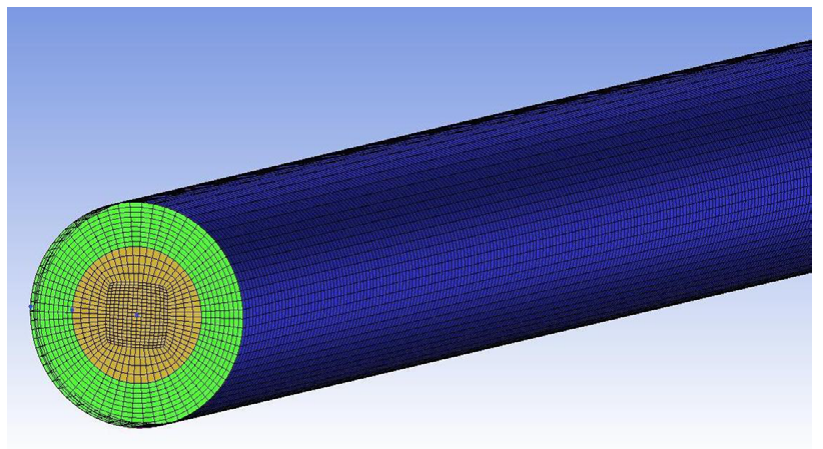

Figure 2: Discretization of the computational domain.

section is discretized by Gauss theorem to convert the volume integral to surface integral. For discretization of the convection term of the yielding governing equations is done by the upwind scheme which ensures boundedness and stability of the solution with an acceptable accuracy. Gauss linear scheme is used for the discretization of the diffusion term. The source term which is considered for the flow in a porous wall is treated explicitly in term of the temporal discretization. The resulting algebraic equations are solved based on the Semi-Implicit Method for Pressure Linked Equations called SIMPLE algorithm [20] to find a converged solution. The under-relaxation factors required to improve the stability of the solution are chosen for the velocity and pressure as 0.3 and 0.7 , respectively. The overall iteration is assumed to be converged when the residual for each equation is below than $10 \mathrm{e}^{-5}$.

\section{Boundary Conditions}

The porous wall is homogeneous, isotropic and characterized by its viscous resistance $\mathrm{D}$ in equation 3 or permeability $\varepsilon$. Moreover, the used fluid water is assumed to be incompressible, isothermal and with a constant density and viscosity of $1000 \mathrm{~kg} / \mathrm{m}$ and $10 \mathrm{e}^{-6} \mathrm{~m}^{2} / \mathrm{s}$, respectively. The flow is laminar since a Reynolds number of less than 1000 is calculated for all the simulated conditions.

For each parameter such as pressure and permeability, a steady state simulation is carried out resulting the steady state flux, pressure drop and axial as well as radial velocity distribution. The boundary conditions are adjusted in such a way; they match the operating conditions of the experiment. 
For all the simulations, the driving force for the water is the pressure gradient. Therefore, the pressure has a constant value at the inlet namely operating pressure and an atmospheric pressure at the outlet. No slip velocity at the dead-end is set which is treated as a wall. The components of the velocity gradient are considered to be zero. Different permeability values for the porous wall are taken into account. This variation of the capillary permeability considers the formation of the fouling layer since this layer has a porous structure.

\section{Results and Discussion}

The flow through the porous wall and inside the capillary membrane operated in dead-end mode was simulated. The operating parameters for the numerical simulation match those used in the experiment in terms of the applied pressure and the capillary permeability. The range of the boundary conditions applied in this numerical simulation is explained in the Table 1.

This range is adapted for the experiments used commonly for the UF in dead-end capillary membrane. The validation of the results is realized by comparing the flux at the outlet between the experimentally measured and simulated values. Figure 3 shows a good agreement between the simulation and the experiment results with very small deviations due to the accuracy of the measurements. Both simulation

\begin{tabular}{|c|c|}
\hline Parameter & Simulated range \\
\hline Permeability [liter $/\left(\mathrm{m}^{2} \mathrm{~h}\right.$ bar $\left.)\right]$ & $80-400$ \\
\hline Pressure $[\mathrm{bar}]$ & $2-4$ \\
\hline
\end{tabular}

Table 1: Range of the studied boundary conditions.

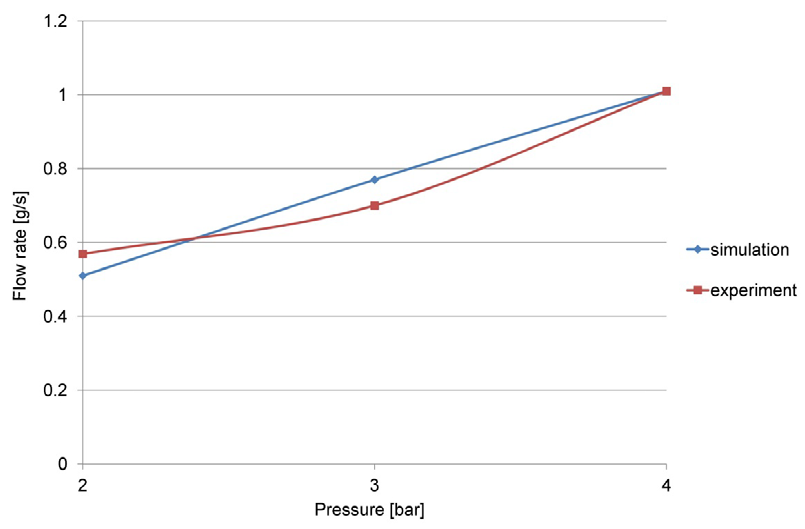

Figure 3: Validation of flow rate.

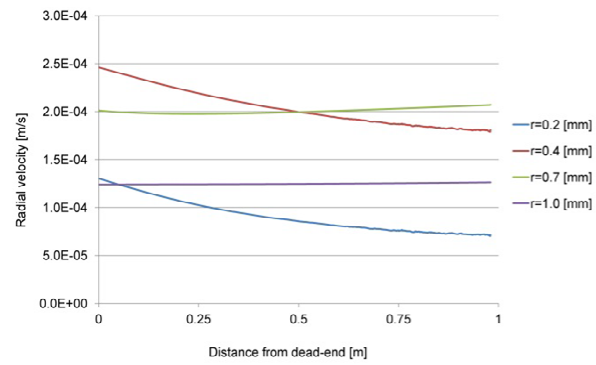

and experiment show that the flow rate is directly proportional to the applied pressure. An increase in the outer pressure on the capillary is accompanied with increase in the water flow rate at the outlet.

Figure 4 shows the axial and radial velocity profiles at laminar flow condition along different lines placed at different distances from the capillary axis. Considering the flow rate balance, the axial velocity increases from zero at the dead-end towards the outlet where the maximum value is reached. For the flow at distance of $r=0.7 \mathrm{~mm}$ along the interface between the porous wall and the capillary surface the axial velocity is very small and refers to negligible tangential variation of the flow in the porous wall. However, the radial velocity at the interface and in the porous wall has a constant value along the capillary. It can be supposed that the velocity in the porous wall is composed only of radial component whereas inside the capillary the dominant component is the radial velocity.

Evaluating the axial velocity at three control lines inserted in different positions across the capillary and porous wall is illustrated in Figure 5. The velocity profile changes with the distance over the capillary length. The fluid accelerates towards the outlet resulting different maximum velocity and subsequently different Reynolds number. The developing velocity profile within the capillary membrane can be observed at the control lines. It also can be seen that the velocity through the porous wall has no axial component. This observation confirms the normal penetrating of the water to the capillary in the presence of the permeate wall. Here $r^{*}$ is in the abscissa which denotes the normalized diameter of the capillary where zero is on the axis. The dimensionless form simplifies the presentation of the results independent of the scale.

The pressure distribution along the three control lines across the capillary is shown in Figure 6. Over the cross section, the pressure in the capillary indicates a constant value whereas in the porous wall this value reaches its maximum.

The effect of various boundary conditions such as the operating pressure and membrane permeability on the flow inside the capillary is studied in the next section.

\section{Effect of the Operating Pressure}

Since the pressure gradient is the driving force for the backwash process, determining the pressure drop inside the capillary gains more interest.

The numerical results show that the pressure changes along the capillary length for various operating pressure. As it is expected, the pressure inside the capillary increases when the applied pressure

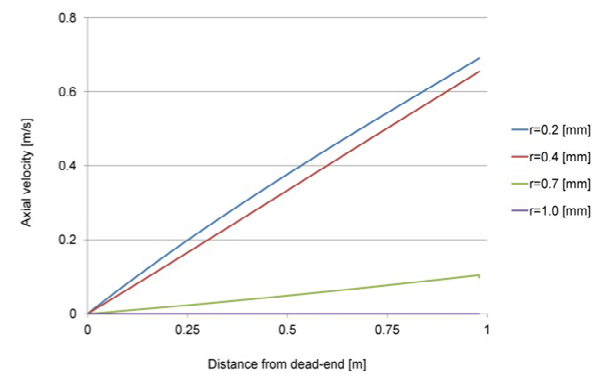

Figure 4: Axial and radial velocity profiles at different control lines along the capillary at 3 [bar] operating pressure. 


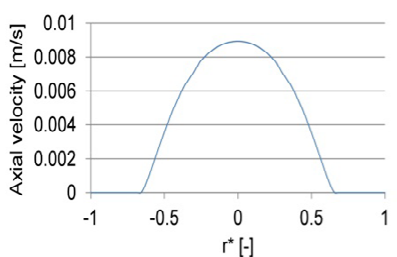

dead-end

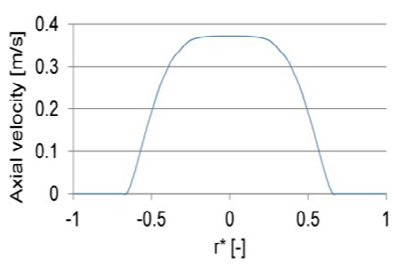

middle

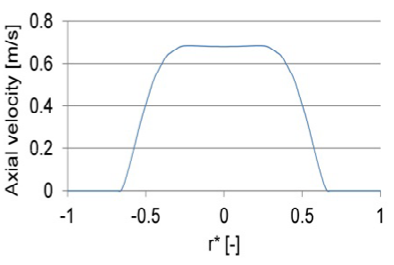

outlet

Figure 5: Axial velocity across the capillary at three control lines, at dead-end, in the middle of the capillary and at the outlet for 3 [bar] operating pressure.

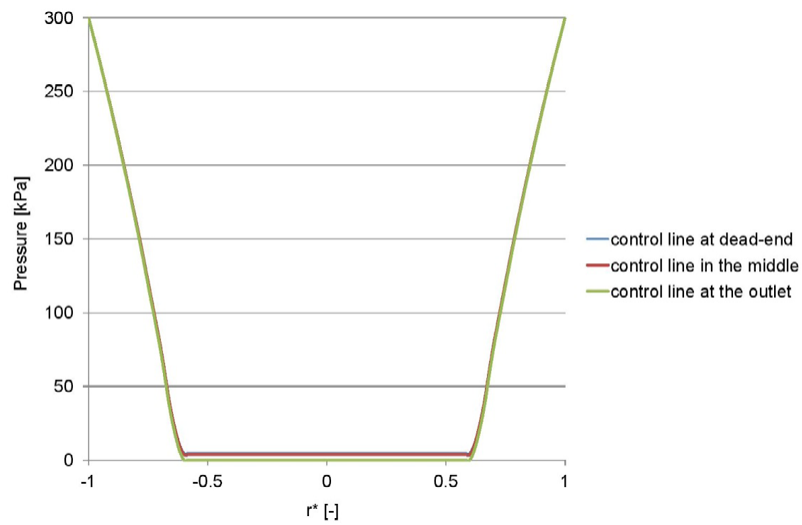

Figure 6: Pressure across the capillary at three control lines, at dead-end, in the middle of the capillary and at the outlet for 3 [bar] operating pressure.

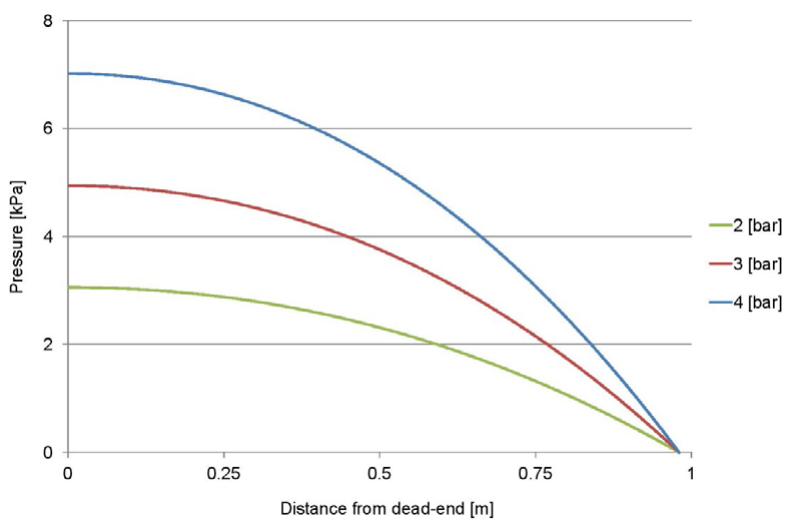

Figure 7: Pressure at the capillary axis for different operating pressure.

increases. The flow rate reduces towards the dead-end, thus increasing the pressure inside the capillary. The pressure starts with value zero at the outlet and increases to its maximum at the dead-end. Increasing the operating pressure improve the removal of suspended particles because the pressure gradient inside the capillary increases that will has consequences on the axial velocity (Figure 7).

At a small operating pressure the Reynolds number indicates laminar flow. However, the flow is fluctuating between laminar and turbulence when the pressure increases. This enhances the energy loss in the capillary and leads to more operating costs.

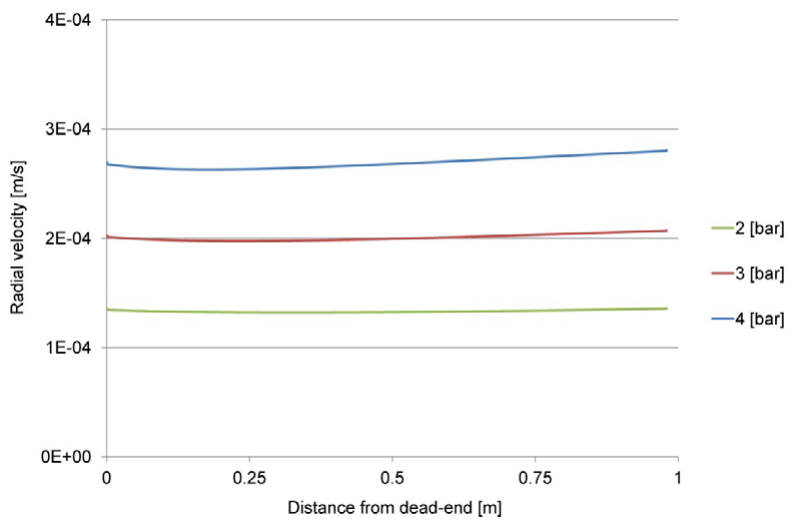

Figure 8: Radial velocity at the interface between porous wall and capillary surface $r=0.7[\mathrm{~mm}]$ for different operating pressure and permeability of 160 [liter/ $\left(\mathrm{m}^{2} \mathrm{~h}\right.$ bar $\left.)\right]$

The radial velocity profile is independent of the operating pressure and has a constant value over the capillary length. In Figure 8 the radial velocity is plotted against the capillary length at the interface between the porous wall and capillary surface $\mathrm{r}=0.7 \mathrm{~mm}$ for different operating pressure. The profile variation from the dead-end to the outlet is very small and the velocity considered as constant. This assumption of uniform normal-wall velocity is found to be a reasonable approach and is applicable for further multiphase simulation when the permeable wall is neglected.

For the axial velocity, increasing the pressure gradient, as referred in Figure 9 leads to significant changes in the driving force which controls flow rate and the axial velocity. Reynolds number recorded from the axial velocity in the capillary at the outlet is 700, 980 and 1260 for 2, 3 and 4 bar operating pressure, respectively.

\section{Effect of the Membrane Permeability}

The fouling layer decreases membrane permeability and forms additional resistance for the water flow through the capillary. The total membrane resistance results from the sum of all involved resistance in series model namely, the membrane resistance, irreversible fouling resistance and cake layer resistance. TTherefore, the thickness of the fouling layer has a significant influence on the pressure drop inside the capillary and the operating pressure. Hereby, the variation of the membrane permeability due to the formation of fouling layer is taken into account.

Table 2 summarizes the flow rate resulting from various vales of the membrane permeability at constant operating pressure. The flow 
Citation: Mansour H, Kowalczyk W (2015) Investigation of Inlet Boundary Conditions on Capillary Membrane with Porous Wall during Dead-End Backwash. J Membra Sci Technol 5: 138. doi:10.4172/2155-9589.1000138

Page 5 of 6

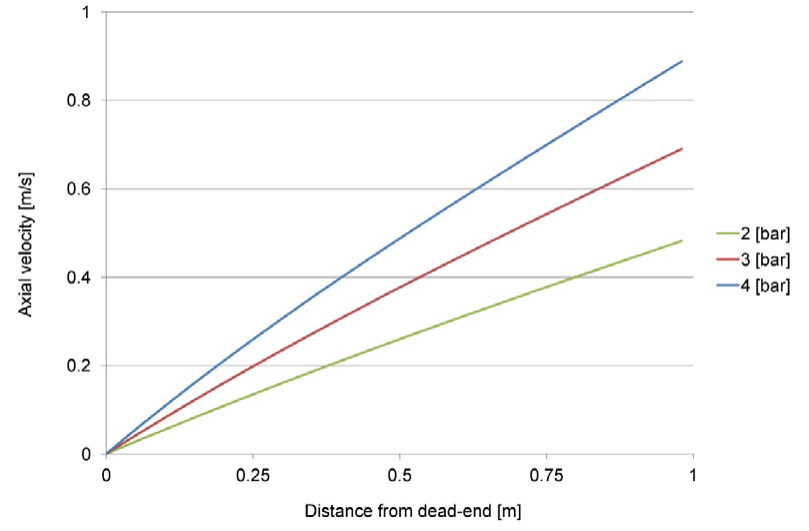

Figure 9: Axial velocity at the capillary axis for different operating pressure and permeability of 160 [liter/ $\left(\mathrm{m}^{2} \mathrm{~h}\right.$ bar $\left.)\right]$

\begin{tabular}{|c|c|}
\hline Permeability [liter/( $\mathbf{m}^{\mathbf{2}} \mathbf{h}$ bar $\left.)\right]$ & Flux $[\mathbf{g} / \mathbf{s}]$ \\
\hline 80 & $3.87 \mathrm{e}-7$ \\
\hline 160 & $7.7 \mathrm{e}-7$ \\
\hline 400 & $1.87 \mathrm{e}-6$ \\
\hline 800 & $3.43 \mathrm{e}-6$ \\
\hline
\end{tabular}

Table 2: Flux at different permeability and 3 [bar] operating pressure.

rate through the porous wall is increasing with the destruction of the fouling layer which changes the resistance towards allowing more water to penetrate the capillary membrane.

In Figure 10, the relation between the fouling layer and the pressure inside the capillary is presented. The pressure and related driving force are higher when the membrane permeability declines. It is assumed that the formation of the fouling layer is a modification of the membrane permeability. Thus, the removal of the deposited particles increases the pressure since it enhances the membrane permeability.

Based on the driving force inside the capillary, the estimated axial velocity at the capillary axis shows a proportional relation to the membrane permeability (Figure 11) the more the membrane permeability, the higher the axial velocity caused by the higher pressure gradient.

For a high permeability, the flow can pass through the porous wall easily and the radial velocity is increasing. However, the profile remains constant over the capillary length apart from the permeability value (Figure 12). These small changes in the radial velocity from the deadend to the outlet have no considerable influence on the flow entering the capillary. Thus, again the assumption of a normal-wall velocity is an acceptable approach and can be used for further simulation without the consideration of the flow in the porous wall.

\section{Conclusions}

The effect of different boundary conditions on the backwash process is investigated. Simulations were carried out for capillary membrane model operated in dead-end mode.

It was found that converting the operating pressure into a constant flux over the capillary length is acceptable approach for the definition of the boundary condition. This assumption has a significant impact on further simulation for the considered model.
The results of the simulations show that, during the backwash water enters the capillary perpendicular to the capillary wall independent of the encountered conditions, such as the fouling layer and operating pressure. Moreover, the membrane permeability has a

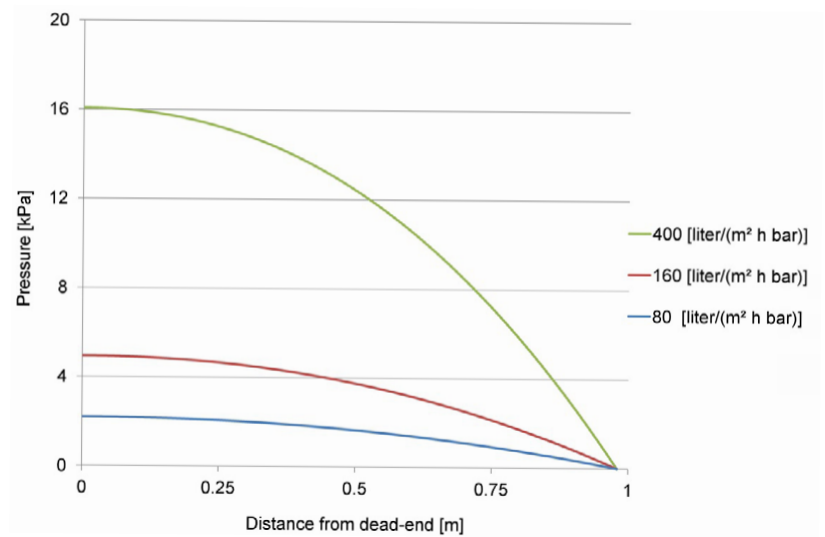

Figure 10: Pressure at the capillary axis for different membrane permeability and 3 [bar] operating pressure.

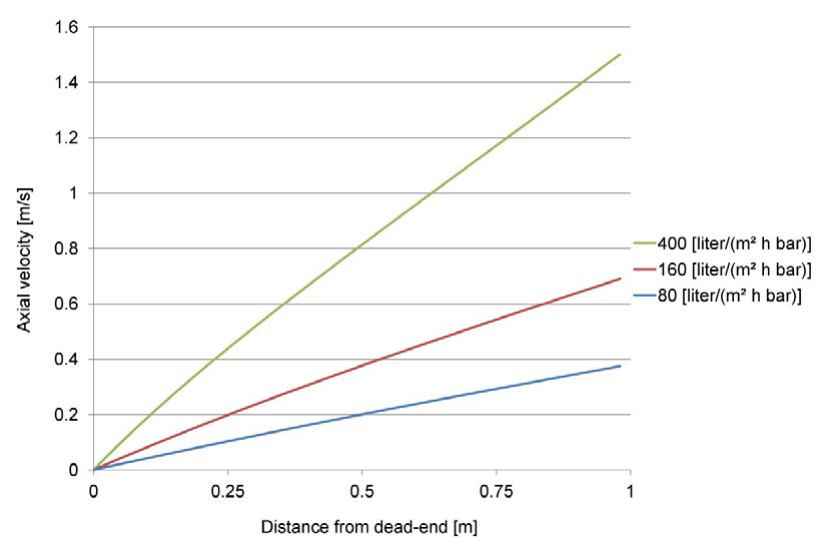

Figure 11: Axial velocity at the capillary axis for different membrane permeability and 3 [bar] operating pressure.

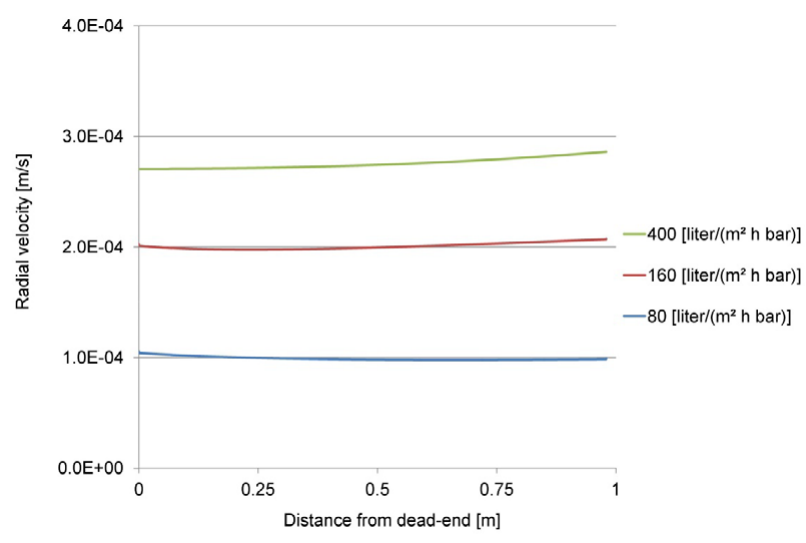

Figure 12: Radial velocity at the interface between the porous wall and capillary surface for different membrane permeability and 3 [bar] operating pressure. 
Citation: Mansour H, Kowalczyk W (2015) Investigation of Inlet Boundary Conditions on Capillary Membrane with Porous Wall during Dead-End Backwash. J Membra Sci Technol 5: 138. doi:10.4172/2155-9589.1000138

Page 6 of 6

proportional relation to the flux penetrating the porous wall, i.e. the flow rate increases when the permeability increases. This permeability represents the packing density of the retained particles on the capillary surface and can accordingly vary. In addition, increasing the operating pressure leads to higher flux at the same membrane permeability. The previously highlighted relation was subsequently confirmed by experimental validation which shows a good agreement with the numerical simulation for predicting the flux at the outlet for different boundary conditions.

\section{Acknowledgements}

The Deutsche Forschungsgemeinschaft (DFG) is gratefully acknowledged for the financial support as well as Professor Rolf Gimbel and Mrs. Anik Kelle from Chair of Process Engineering and Water Technology, University of DuisburgEssen for the experimental validation.

\section{References}

1. Berman AS (1953) Laminar flow in channels with porous walls. Journal of Applied Physics 24: 1232-1235.

2. Yuan SW (1955) Laminar Pipe Flow with Injection and Suction Through a Porous Wall. Trans. ASME 78: 719-724.

3. Bernales B, Haldenwang P (2014) Laminar flow analysis in a pipe with locally pressure-dependent leakage through the wall. European Journal of Mechanics, B/Fluids 43: 100-109.

4. Quaile JP, Levy EK (1975) Laminar Flow in a Porous Tube With Suction. Journal of Heat Transfer 97: 66-71.

5. Terrill RM, Thomas PW (1969) On laminar flow through a uniformly porous pipe. Applied Scientific Research 21: 37-67.

6. Terrill RM (1983) Laminar Flow in a Porous Tube. Journal of Fluids Engineering Transactions of the ASME 105: 303-307.

7. Terrill RM, Shrestha GM (1966) Laminar flow through a channel with uniformly porous walls of different permeability. Applied Scientific Research 15: 440-468.
8. Galowin LS, Fletcher LS, Desantis MJ (1974) Investigation of Laminar Flow in a Porous Pipe with Variable Wall Suction. AIAA Journal 12: 1585-1589.

9. Karode S (2001) Laminar flow in channels with porous walls. Journal of Membrane Science 191: 237-241.

10. Kim AS, Lee YT (2011) Laminar flow with injection through a long dead-end cylindrical porous tube: Application to a hollow fiber membrane. AIChE Journal 57: 1997-2006.

11. Oxarango L, Schmitz P, Quintard M (2004) Laminar flow in channels with wall suction or injection: a new model to study multi-channel filtration systems. Chemical Engineering Science 59: 1039-1051.

12. Hanspal NS, Waghode AN, Nassehi V, Wakeman RJ (2009) Development of a predictive mathematical model for coupled stokes/Darcy flows in cross-flow membrane filtration. Chemical Engineering Journal 149: 132-142.

13. Nassehi V (1998) Modelling of combined Navier-Stokes and Darcy flows in crossflow membrane filtration. Chemical Engineering Science 53: 1253-1265.

14. Tilton N, Martinand D, Serre E, Lueptow RM (2012) Incorporating Darcy's law for pure solvent flow through porous tubes: Asymptotic solution and numerical simulations. AIChE Journal 58: 2030-2044.

15. Teng H, Zhao TS (2000) An extension of Darcy's law to non-Stokes flow in porous media. Chemical Engineering Science 55: 2727-2735.

16. Wiley DE, Fletcher DF (2003) Techniques for computational fluid dynamics modelling of flow in membrane channels. Journal of Membrane Science 211 : 127-137.

17. Pak A, Mohammadi T, Hosseinalipour SM, Allahdini V (2008) CFD modeling of porous membranes. Desalination 222: 482-488.

18. Ghidossi R, Daurelle JV, Veyret D, Moulin P (2006) Simplified CFD approach of a hollow fiber ultrafiltration system. Chemical Engineering Journal 123: 117-125.

19. Forchheimer $P$ (1901) Wasserbewegung durch Boden. Zeitschrift des Vereines Deutscher Ingenieuer 45: 1782-1788.

20. Ferziger JH, Peric M (2002) Computational Methods for Fluid Dynamics Springer London, Limited. 